\title{
Role of hysteretic damping in the earthquake response of ground
}

\author{
N. Yoshida \\ Tohoku Gakuin University, Japan
}

\begin{abstract}
Parametric studies are carried out to investigate the role of the hysteretic damping in earthquake response analysis. The maximum acceleration is found to have an upper bound under very large earthquakes and hysteretic damping does not affect it. On the other hand, response acceleration becomes large in the shorter period as the hysteretic damping increases. It comes because stiffness after the unloading becomes large so as to increase the hysteretic damping ratio, which is a quite different feature that viscous damping has although they are usually understood to have the same mechanical nature.
\end{abstract}

Keywords: earthquake, hysteretic damping, shear strength, upper bound acceleration.

\section{Introduction}

Nonlinear behaviour of soils for the earthquake response analysis of ground is usually defined by strain dependent shear modulus and damping ratio. As such, damping ratio is supposed to be an important mechanical property. It has been supposed to supress the earthquake response; earthquake motion becomes smaller as the damping ratio increases.

On the other hand, the authors showed a different point of view [1]. Maximum acceleration does not exceed the upper bound acceleration under the large ground motion. It means that damping ratio does not work so as to supress the ground acceleration at the ground surface. Instead, another aspect is found; response at high frequency is excited as the damping ratio.

In the previous study, however, only one example was shown. A series of parametric study is carried out in this paper in order to see what happens when the hysteretic damping changes. 


\section{Constitutive models}

Three stress-strain models were used in this study, which are called Hyperbolic, $\mathrm{H}-\mathrm{D}$, and H-D/w E. All models use the same hyperbolic model for the skeleton or backbone curve,

$$
\tau=\frac{G_{0} \gamma}{1+\gamma / \gamma_{r}}
$$

where $\tau$ and $\gamma$ denote shear stress and shear strain, respectively, $G_{0}$ denotes elastic shear modulus, and $\gamma_{r}$ denotes reference strain. The hysteresis curves are defined differently as follows

1) Hyperbolic: ordinary hyperbolic model whose hysteresis loop is developed by applying the Masing's rule to a skeleton curve.

2) H-D: damping characteristics is evaluated from the proposal by Hardin and Drnevich [2],

$$
h=h_{\max }\left(1-G / G_{0}\right)
$$

where $G$ denotes shear modulus and $h_{\max }$ is maximum damping ratio. The hysteresis curve that satisfies this equation can be made by using the method proposed by the authors [3]. The hyperbolic equation same with Eq. (1) is used for the hysteresis curve in this method, but two parameters $G_{0}$ and $\gamma_{r}$ do not have mechanical meaning because they are automatically evaluated in order to get damping ratio defined as Eq. (2).

3) H-D/w E: many constitutive models assume that stiffness at unload is same with the initial or elastic modulus, but, as shown in Figure 1, it decreases with strain amplitude as

$$
\frac{G_{0}}{G_{\max }}=\frac{1-G_{\min } / G_{0}}{1+\gamma / \gamma_{r 0}}+\frac{G_{\min }}{G_{0}}
$$

where $G_{\text {min }}$ denotes minimum stiffness at unloading. The hysteresis curve that satisfy Eq. (3) is obtained by the previous method, but the Ramberg-Osgood model is used in order to add the new condition, Eq. (3).
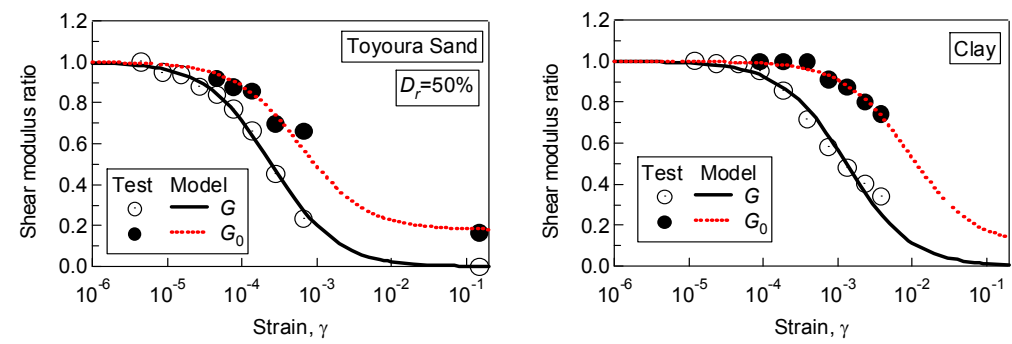

Figure 1: $\quad$ Shear modulus and stiffness at unloading as a function with respect to strain. 


\section{Brief review of previous calculation}

\subsection{Soil profiles and material}

Soil in the Tokyo city area [4] shown in Figure 2 was analysed. Here, $V_{s}$ denotes $\mathrm{S}$ wave velocity, $\rho$ denotes density, $c$ denotes cohesion, and $\phi$ denotes internal friction angle. Model parameters are set as in Table 1.

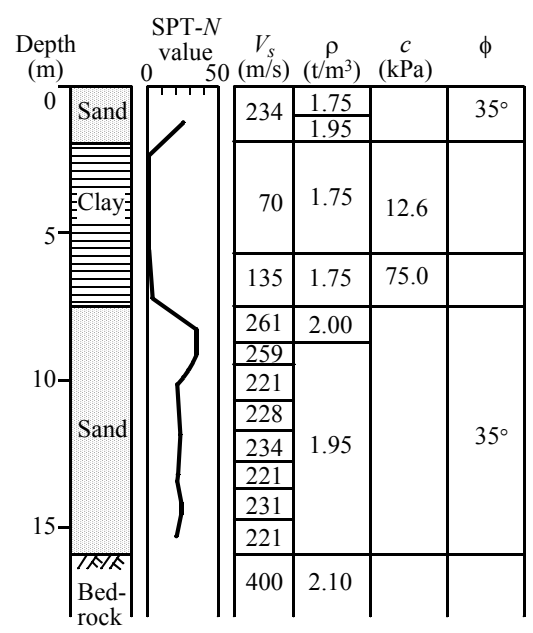

Figure 2: $\quad$ Soil profiles.

Table 1: $\quad$ Model parameters.

\begin{tabular}{ccccc}
\hline Material & $\gamma_{r}$ & $h_{\max }$ & $\gamma_{r 0}$ & $G_{\min } / G_{0}$ \\
\hline Sand & $8.63 \times 10^{-4}$ & 0.22 & 0.002 & 0.4 \\
Clay & $1.42 \times 10^{-3}$ & 0.22 & 0.013 & 0.1 \\
\hline
\end{tabular}
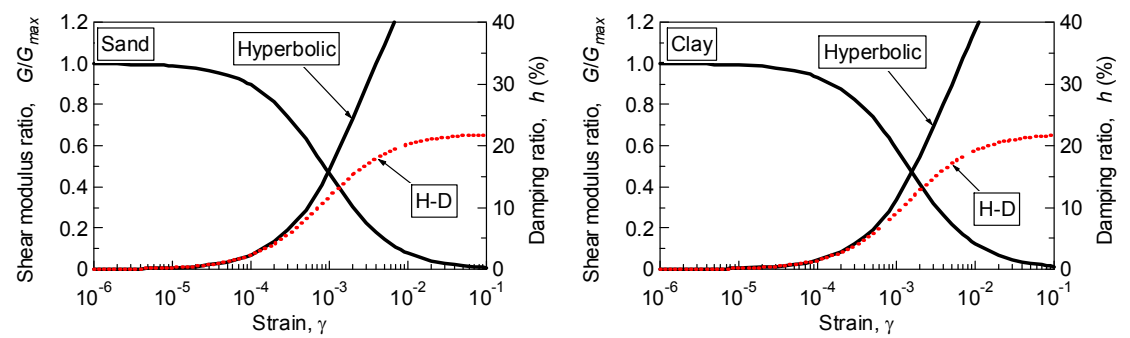

Figure 3: Dynamic deformation characteristics of sand and clay.

Cyclic deformation characteristics are shown in Figure 3. Here, both H-D and H-D/E show the same damping characteristics and, as well known, the hyperbolic model shows larger damping ratio at large strains. Stress-strain curve 
is shown in Figure 4 with shear strain amplitude of $0.6 \%$ and $4 \%$, respectively. Curves are quite different between the hyperbolic model and two H-D type models, but those by the two H-D type models are similar to each other.
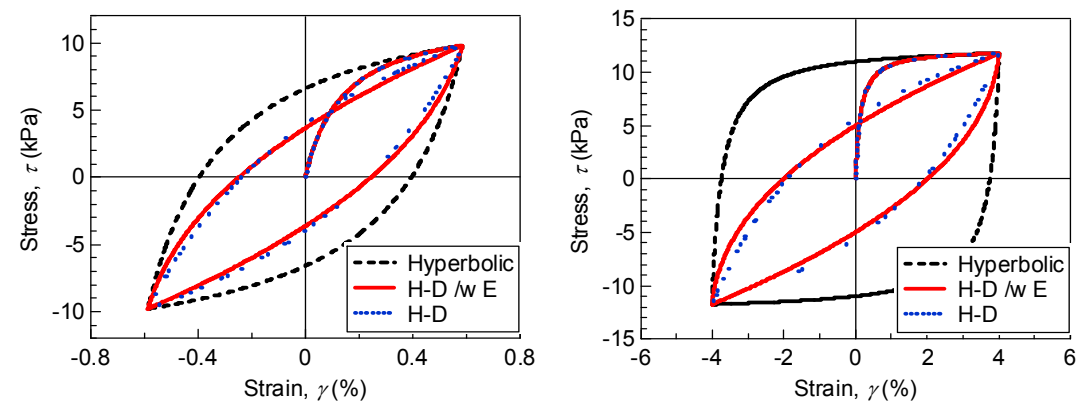

Figure 4: $\quad$ Stress-strain curve of clay.

\subsection{Earthquake motions}

Two earthquake motions are chosen among the earthquake motions shown in ref. [4]. The one has limited number of large amplitude waves and the other large number of large amplitude waves; they are called the shock wave and the vibration wave, respectively. Among them, only the result by using the shock wave is shown in this paper. Since large strain behavior is interested, the acceleration is increased so that the peak acceleration becomes $8 \mathrm{~m} / \mathrm{s}^{2}$ at the outcrop base layer. The waveform is shown in Figure 5.

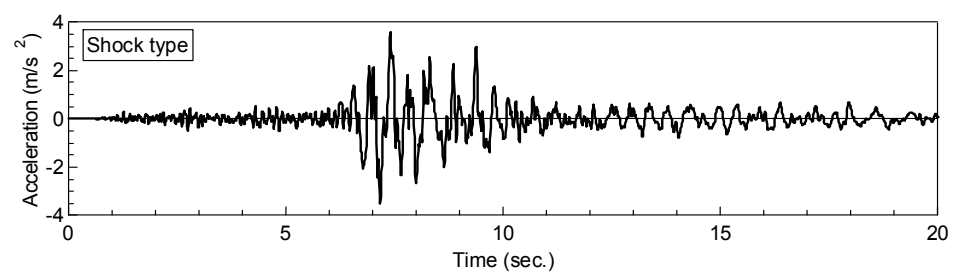

Figure 5: $\quad$ Earthquake motions to the engineering seismic base layer.

\subsection{Response under shock wave and discussion}

Maximum response is shown in Figure 6. The maximum acceleration decreases rapidly from GL-5.8 m, resulting in about $2 \mathrm{~m} / \mathrm{s}^{2}$ at the ground surface. Since the layer between GL-2.8 and $5.8 \mathrm{~m}$ (clay layer) shows large strains up to several percent, shear stress in these layer reaches nearly the shear strength, which can be confirmed through the chained line (shear strength) in Figure 6. If a layer reaches shear strength, acceleration above this layer reaches limit acceleration 
$\alpha_{u l t}$ [5] because of the equilibrium condition between the shear stress in this layer and the inertia force above this layer, as

$$
\alpha_{u l t}=\tau_{f} G / \sigma_{v}
$$

where $\tau_{f}$ denotes shear strength of the key layer, $G$ denotes acceleration of gravity and $\sigma_{v}$ denotes overburden stress. Applying this equilibrium condition into the 4th layer (GL-2.8 to $3.8 \mathrm{~m}$ ), expected upper bound acceleration becomes $2.08 \mathrm{~m} / \mathrm{s}$, which agrees with the maximum acceleration at the ground surface in Figure 6. It is emphasized that damping ratios are quite different in these three cases, but the difference cannot be seen in the maximum acceleration.

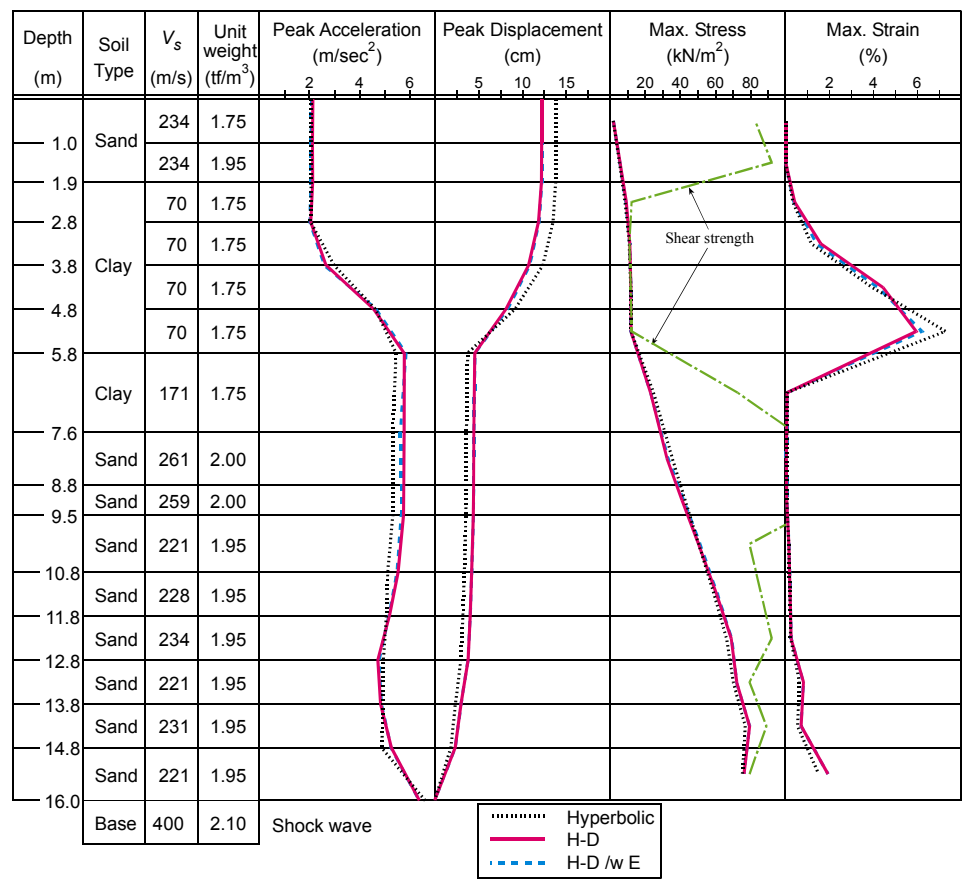

Figure 6: $\quad$ Maximum response under shock wave.

Response spectra are computed from the acceleration at the ground surface, which is shown in Figure 7. There is no significant difference at the period longer than about $0.5 \mathrm{~s}$, but the hyperbolic model shows much larger accelerations than the other two cases in the shorter period. It is emphasized that the damping ratio is the largest in the hyperbolic model. If large damping ratio suppresses the vibration, response acceleration by the hyperbolic model must be smaller than other two cases.

It is also noted that two H-D models (H-D and H-D/ E) shows almost similar response. It indicates that stiffness at unload is not a big issue. The hysteresis loops essentially become similar shape because they are spindle shape with the same area. 


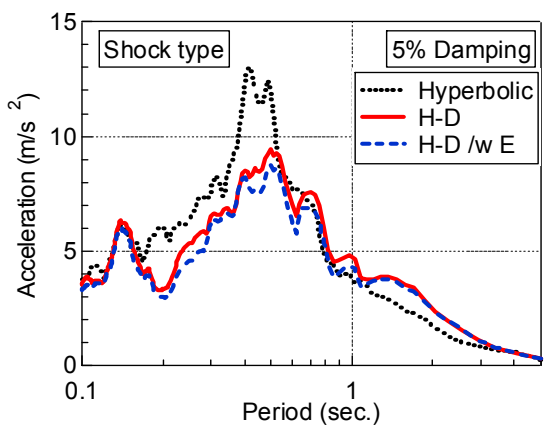

Figure 7: $\quad$ Response spectrum under shock wave.

The stress-strain curves at the 6th layer where shear strain becomes largest are shown in Figure 8. Apparent stiffness after the unloading seems much larger in the hyperbolic model than those in the two H-D models. As seen in Figure 4, stiffness after unloading should be kept large so as to keep large damping ratio in the hyperbolic model. On the other hand, as damping ratio is small in the two $\mathrm{H}-$ $\mathrm{D}$ models, stiffness after the unloading is smaller than that of the hyperbolic model. Therefore, apparent stiffness is larger in the hyperbolic model than in the two H-D models. This is the reason why the hyperbolic model shows larger response acceleration than the two H-D models.
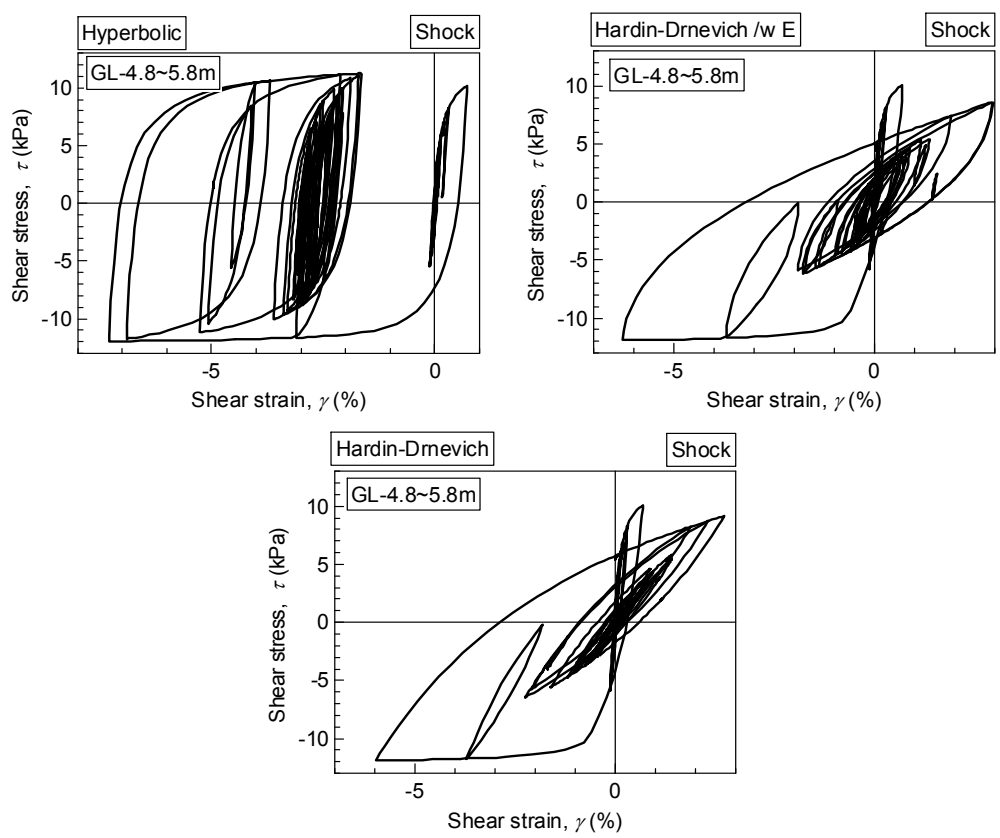

Figure 8: $\quad$ Stress-strain curves at 6th layer under shock wave. 
Although result under shock type acceleration is introduced here, the result under the vibration type earthquake also shows similar behaviour.

\section{Effect of input acceleration}

If upper bound acceleration associated with the small shear strength of soil, amplification depends on the input acceleration. Then, a parametric study is carried out to see the effect of the magnitude of the input motion to the earthquake response. The same soil profile and the input earthquake motion are used in the parametric study except that the maximum damping ratio $h_{\max }$ is set $25 \%$. The magnitude of the input earthquake is scaled and applied. The input motion used in the previous section is used as standard value; magnification factor is the ratio from this input motion.

Figure 9 shows maximum response under the magnification factors $0.01,0.1$, $0.5,1.0,1.5$, and 2.0. When the input acceleration is very small, maximum acceleration increases upward. As the input acceleration increases, however, it becomes constant in certain thickness. For example, maximum acceleration is nearly constant between GL and GL-3.8 m and between GL-5.8 m and $12.8 \mathrm{~m}$. Just below these layers, maximum acceleration decrease rapidly. Shear strains in these layers are more than $1 \%$, which indicates that shear stresses reach near the shear strength (see Figure 8). It means that these constant maximum accelerations are upper bound acceleration. There are two key layers defining the

\begin{tabular}{|c|c|c|c|c|c|c|c|c|}
\hline Depth & $\begin{array}{l}\text { Soil } \\
\text { Type }\end{array}$ & $\begin{array}{c}V_{s} \\
(\mathrm{~m} / \mathrm{s})\end{array}$ & Density & $\begin{array}{l}\text { Max. Acceleration } \\
\left(\mathrm{m} / \mathrm{sec}^{2}\right)\end{array}$ & Max. I & $\begin{array}{l}\text { Displacement } \\
(\mathrm{cm})\end{array}$ & $\begin{array}{c}\text { Max. Stress } \\
\left(\mathrm{kN} / \mathrm{m}^{2}\right)\end{array}$ & $\begin{array}{c}\text { Max. Strain } \\
(\%)\end{array}$ \\
\hline \multirow{3}{*}{\begin{tabular}{|l}
-1.0 \\
\end{tabular}} & & & & & & $=$ & & \\
\hline & \multirow{2}{*}{ Sand } & 234 & 1.75 & & & I & & \\
\hline & & 234 & \begin{tabular}{|l}
1.95 \\
\end{tabular} & & & i & & \\
\hline \multirow{2}{*}{$\begin{array}{r}-1.9 \\
-2.8\end{array}$} & \multirow{4}{*}{ Clay } & 70 & 1.75 & 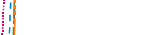 & & i & & \\
\hline & & 70 & 1.75 & ii) & & 1 & & \\
\hline \multirow{2}{*}{$\begin{array}{r}3.8 \\
-4.8\end{array}$} & & 70 & 1.75 & & & & & \\
\hline & & 70 & 1.75 & & & & & $\bar{y}$ \\
\hline-5.8 & Clay & 171 & 1.75 & il & & & & \\
\hline \begin{tabular}{|l}
-7.6 \\
\end{tabular} & Sand & 261 & 2.00 & ii & & & & \\
\hline \multirow{2}{*}{$\begin{array}{r} \\
\end{array} 9.8$} & Sand & 259 & 2.00 & 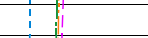 & & & & \\
\hline & Sand & 221 & 1.95 & il & 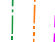 & & & \\
\hline-10.8 & Sand & 228 & 1.95 & i & 1 & & 111 & \\
\hline \multirow{2}{*}{\begin{tabular}{|r}
11.8 \\
-12.8
\end{tabular}} & Sand & 234 & 1.95 & $i_{1}$ & & Mas factor & ¿ ? & \\
\hline & Sand & 221 & 1.95 & $d_{1}^{T}$ & 11 & 0.01 & if & 1 \\
\hline \multirow{2}{*}{$\begin{array}{r}-13.8 \\
-14.8\end{array}$} & Sand & 231 & 1.95 & 1 & 1 & ----0.5 & i) & 11 \\
\hline & Sand & 221 & 1.95 & & & $\begin{array}{l}--1.5 \\
----2.0\end{array}$ & : & 111 \\
\hline-16.0 & Base & 400 & 2.10 & & & & & \\
\hline
\end{tabular}

Figure 9: $\quad$ Maximum response. 
upper bound acceleration, both of which control maximum accelerations above these layers. The shear strength of the upper layer is smaller than that of the lower layer.

Figure 10 shows change of the maximum acceleration at the ground surface when different magnitude of the earthquake works, and Figure 11 shows amplification factor (maximum acceleration at the ground surface / maximum acceleration of the input motion). Maximum acceleration increases as the input motion becomes large, but rate of the increase gradually decreases, resulting in constant maximum acceleration or upper bound acceleration. This feature is same with the one by Idriss [6] and Suetomi and Yoshida [7]. In the same manner, the amplification factor is 2.35 under the very small input or elastic response, but it decreases quickly as nonlinear behaviour becomes significant. It is noted that the upper bound acceleration depends on the shear strength of the weakest layer and its depth, relationship in Figure 10 is not a unique curve but depends on the site.

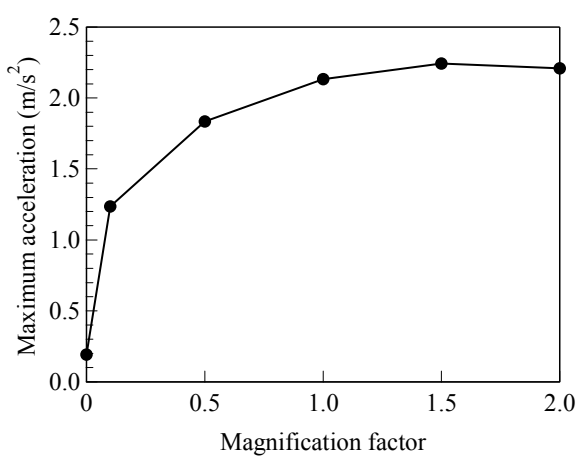

Figure 10: Maximum acceleration vs. input motion.

As described above, this behaviour is first found by Idriss [6], but the mechanism is not known at that time. Through this study, it becomes clear that it is caused because there is upper bound acceleration.

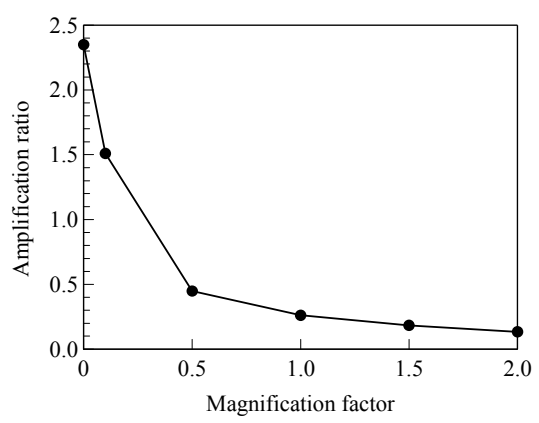

Figure 11: Amplification ratio vs. input motion. 


\section{Effect of hysteretic damping}

The maximum damping ratio $h_{\max }$ is chosen a parameter in this section. The theoretical maximum value of $h_{\max }$ is $2 / \pi$. Then $h_{\max }$ is varied from $10 \%$ to $60 \%$.

Figure 12 shows maximum response. The maximum acceleration at the ground surface is nearly constant regardless of the $h_{\max }$. Therefore, it is clear that the damping ratio does not work to suppress the earthquake motion.

Figure 13 shows maximum acceleration vs. $h_{\max }$ relationships. The maximum acceleration at the ground surface seems constant in Figure 12, but it decreases

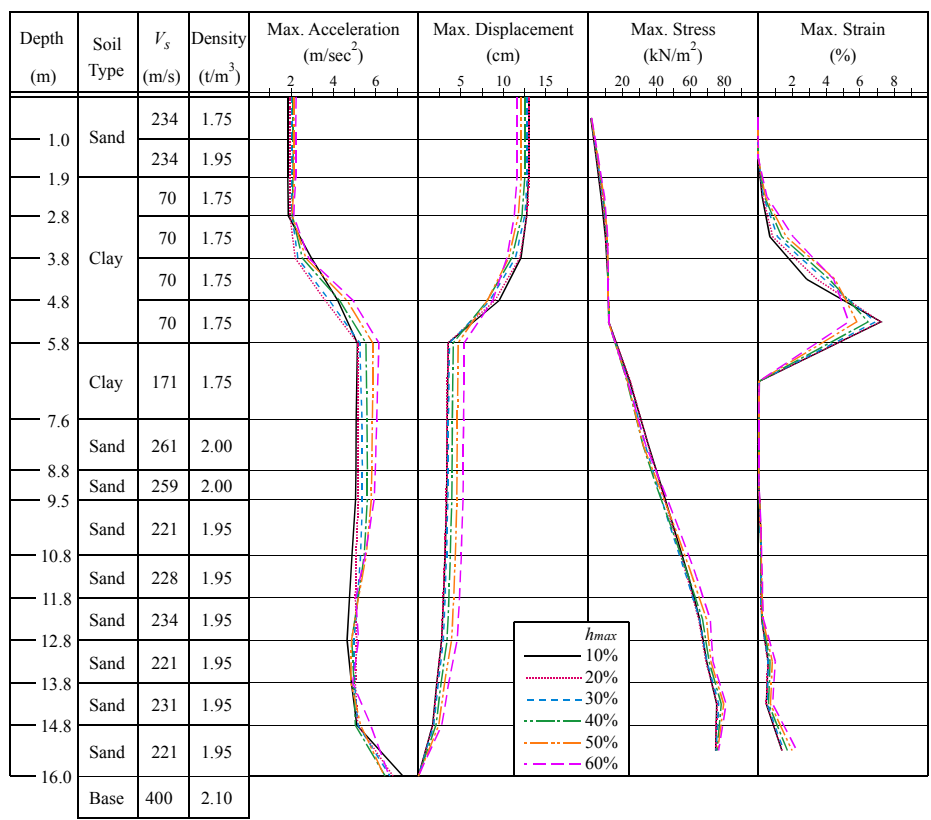

Figure 12: $\quad$ Maximum response.

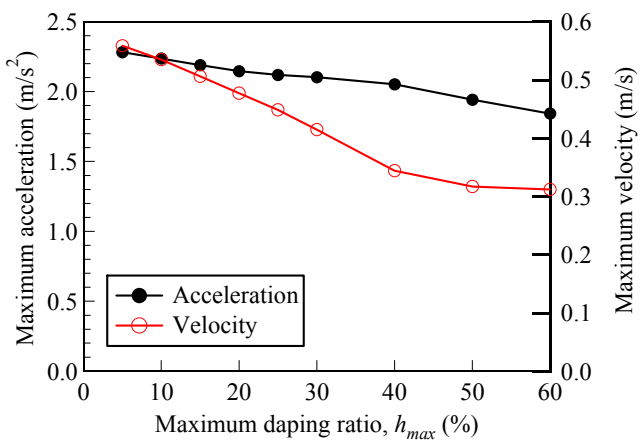

Figure 13: Maximum responses vs. maximum damping ratio. 
as $h_{\max }$. Then, it seems that $h_{\max }$ works to suppress the response of the ground. However, when looking at Figure 12 in detail, different feature can be seen.

Maximum strains below GL-2.8 $\mathrm{m}$ increases as $h_{\max }$ increases. In indicates that key layer that controls the upper bound acceleration goes downward. As can be seen from Eq. (4), the upper bound acceleration decreases as the depth of the key layer increases or $\sigma_{v}$ increases.

Maximum damping ratio $h_{\max }$ is usually between 15 and 25 degrees in the actual soil. In this damping ratio, change of the maximum acceleration is not large. In this meaning, damping ratio is said not to affect the maximum acceleration at the ground surface.

Figure 14 shows acceleration response spectra under the damping ratio of 5\%. The response acceleration becomes larger in shorter period as $h_{\max }$ increases. On the other hand, it becomes smaller in longer period. The boundary is around $0.5 \mathrm{~s}$. It is noted that shorter period less than $0.5 \mathrm{~s}$ is very important period in many building or civil engineering structures. From the discussion above, it is clear that this large response acceleration occur as the stiffness after the unloading becomes larger as $h_{\max }$ increases. This is quite different feature that the word "damping" has.

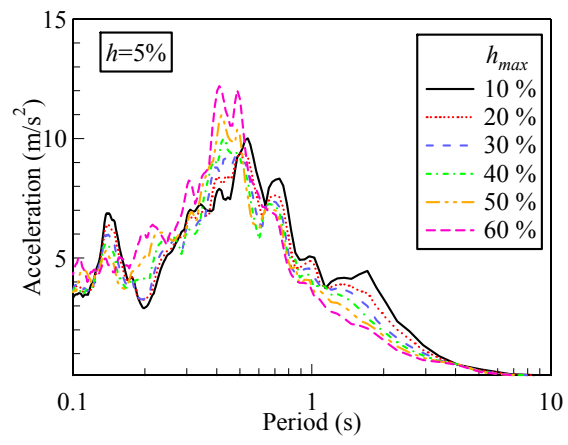

Figure 14: Acceleration response spectra.

\section{Conclusion}

Parametric study is carried out in order to see the mechanism how hysteretic damping works in the earthquake response of the ground. The following conclusions are obtained.

1) Large hysteretic damping does not imply small response or amplification under the large earthquake motion because maximum acceleration has upper bound associated from the failure of the weak layers.

2) Stiffness after the unloading becomes large as the damping ratio increase because area of the hysteresis loop must be large. This large stiffness excites high frequency or small period ground shaking, resulting in large response acceleration in the small period range. 
The second conclusion is quite different feature that the term "damping" has. In this sense, hysteresis damping does not have the same mechanical property with the viscous damping.

\section{References}

[1] Yoshida, N. (2009): Effect of hysteretic damping and stiffness at unloading on response of ground during earthquake, Proc., 3rd Greece-Japan Workshop on seismic design, observation, retrofit of foundations, Santorini, Greece, pp. 573-583

[2] Hardin, B. O. and Drnevich, V. P. (1972): Shear modulus and damping in soils: design equations and curves, Proc. of the American Society of civil engineers, Vol. 98, No. SM7, pp. 667-692

[3] Ishihara, K., Yoshida, N. and Tsujino, S. (1985): Modelling of stress-strain relations of soils in cyclic loading, Proc. 5th International Conference for Numerical Method in Geomechanics, Nagoya, Vol. 1, pp. 373-380, 1985

[4] Sato, M., Yasuda, S., Yoshida, N. and Masuda, T. (1998): Simplified method for estimating maximum shear stress in the ground during earthquakes, Journal of Geotechnical Engineering, Proc. JSCE, No. 610/III45, pp. 83-96 (in Japanese)

[5] Suetomi, I., Sawada, S., Yoshida, N. and Toki, K. (2000): Relation between shear strength of soil and upper limit of earthquake ground motion, Jour. of Structural Mechanics and Earthquake Engineering, Proc. JSCE, No. 654/I52, pp. 195-206 (in Japanese)

[6] Idriss, I. M. (1990): Response of Soft Soil Sites during Earthquakes, Proceedings, H. Bolton Seed Memorial Symposium, Berkeley, California, Vol. 2, pp. 273-289

[7] Suetomi, I. and Yoshida, N. (1998): Nonlinear behavior of surface deposit during the 1995 Hyogoken-nambu earthquake, Soils and Foundations, Special Issue on Geotechnical Aspects of the January 171995 HyogokenNambu earthquake, No. 2, pp. 11-22 